\title{
LED Backlight for Better Accuracy in Medical Imaging
}

\author{
Silvio Bonfiglio and Luigi Albani \\ FIMI Philips, via S. Banfi 1, 21047 Saronno, Italy \\ \{Silvio.Bonfiglio, Luigi.Albani\}@philips.com
}

\begin{abstract}
In clinical tasks the display is often the natural interface between the medical system and the medical professionals and in the current image-centric healthcare the accuracy of the visualized images represents a key requirement; ideally no compromise would be acceptable. In the recent past LED backlights for liquid-crystal displays have been intensively investigated for their use in displays addressed to the mainstream markets (mobile and portable displays, computer displays and TV). Accordingly adapted, they could offer new opportunities also to the displays used in healthcare by allowing better accuracy and consistency of the medical images. In this respect they could make possible a new, important advance towards a better quality of care.In this paper we will describe a novel LED backlight solution suitable for medical imaging.
\end{abstract}

Keywords: BLU, backlight, backlight unit, color gamut, display, healthcare, LCD, LED, imaging, image accuracy, medical imaging.

\section{Introduction}

Imaging in healthcare is opening new horizons in the continuous effort of the medical science towards a better quality of care. Images of parts of our body are used for diagnostics, to detect as earlier as possible the uprising of pathologies. Images - often in 3D format - are used for the planning of complex surgical interventions and minimally invasive, image-guided procedures are replacing traditional surgical approaches. Images are transmitted from one part to another one of the world and telemedicine and telesurgery are removing the physical distances between the patient and the physician or the surgeon. Even in therapeutic treatments the images are becoming very important: think about the virtual reality used in therapeutic programs for patients suffering from psychological stress or other mental diseases. Other new and innovative display technologies are entering into the clinical setting: large screen displays and projectors for multi-image applications, 3D displays in ultrasound and in CT or MRI, headmounted displays for augmented reality and for immersive virtual reality experiences, portable displays for the mobile point of care, etc.

The display visualizing all these images represents the "natural interface" between the medical system and the physician. Since each detail of the image could have a "clinical relevance", the overall image chain will be effective if the display reproduces the "source image" without introducing artifacts and without reducing the quantity of "clinical information" it contains and if there is consistency over time and between same images reproduced in different displays. 
Currently the display represents one of the weakest element in the medical image chain:

- spatial (dot/inch) and grayscale (number of displayed gray levels) resolutions are below the characteristics of some medical images (e.g. mammography) and below the capability of the human vision system,

- color gamut i.e. the overall range of reproduced colors is often limited and far below the overall range of available colors,

- response time, viewing angle and contrast in some displays (e.g. the liquid-crystal displays) are sub-optimal and these limitations introduce unwanted artifacts,

- brightness is often limited to avoid excessive power dissipation (e.g. in LCDs) or visual artifacts.

The backlight of the LCD monitors has been an area heavily investigated in the last decade.

In the transmissive displays such as the liquid-crystal ones the backlight represents the source of light that each LC cell - after modulating it in intensity according to the applied input video signal - will transfer outside (see fig. 1).

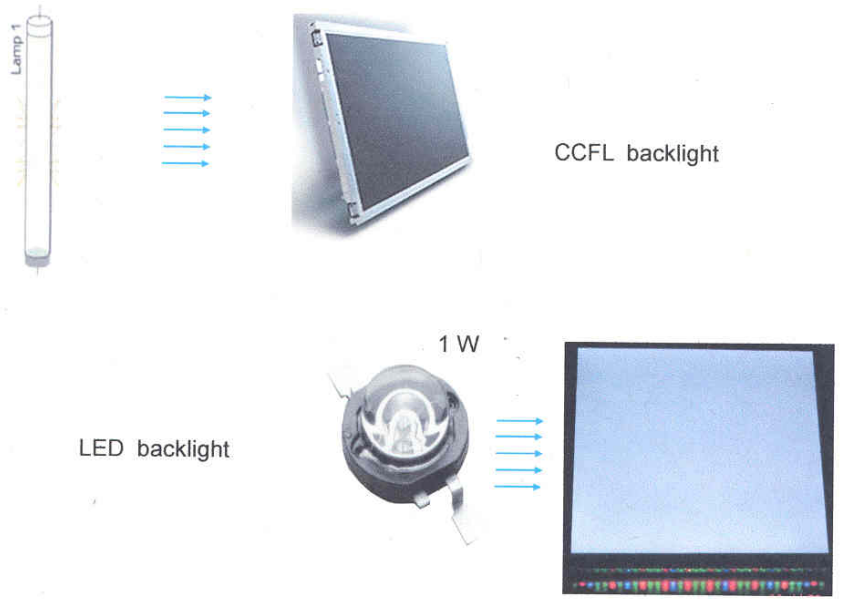

Fig. 1. CCFL and LED backlights

For long time LCD backlights have been based on the use of fluorescent lamps (cold cathode fluorescent lamps - CCFL); among the alternative technologies developed in the recent past LED backlights have been the most successful ones and are expected to replace the traditional CCFL in the coming years in all the applications (from cars and traffic lights to mobile phones, monitors and TVs) and at the same time to enter in the general lighting market.

\section{LED Backlights - General Benefits}

The list of benefits offered by LED backlights is quite long: 
- Wider gamut: $105 \%$ of the NTSC color space compared to $65-75 \%$ of the conventional CCFL technology ; this extended color gamut allows a richer variety of colors including vivid and saturated true colors ;

- Real-Time Color Management - Instantaneously controlling of colors on a variety of data streams to enhance viewing experience;

- Fully dimmable without color variation;

- Fast response time allowing the implementation of blinking backlights for larger contrast and greater picture clarity (less blurring);

- Instant on (less than 100ns) (@ full color, 100\% light);

- Higher energy efficiency;

- Very Long Operating Life (up to100k hours) and increased durability and reliability of system;

- No UV / IR in the light source (only visible wavelengths emitted) avoiding degradation of the optical system and extending product life;

- Green/Environmental : no mercury in system;

- Safe operation - low voltage.

Moreover it is important to highlight that the tuning of the white point - when using a conventional CCFL backlight - is possible only by acting on the R,G,B input video signals with the consequent drawback of a reduction of the driving levels (smaller dynamics). On the contrary in LED backlights the tuning is done by acting on the primary colors of the LEDs and the same number of reproducible colours at various colour temperatures can be achieved (see Fig. 2).

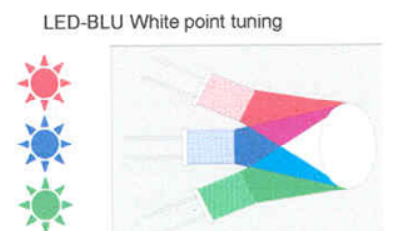

LED Backlight

Same number of reproducible colour at various Colour temp

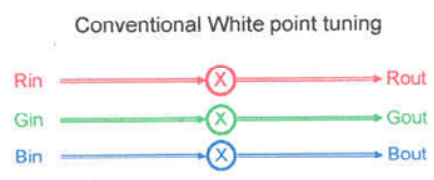

CCFL Backlight

Reduced Driving Levels

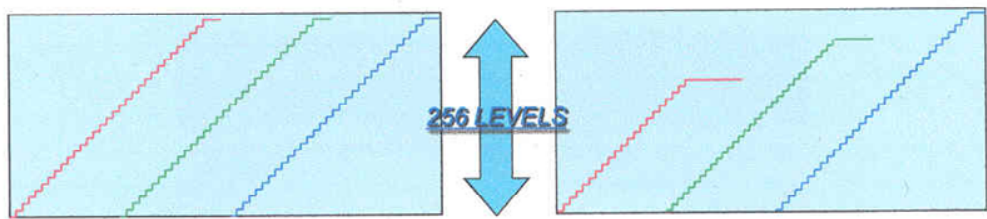

Fig. 2. White point tuning in LED and in CCFL backlights

Through the use of LED backlight the display will enrich its color gamut and enhance the effectiveness of the interfacing between the electronic equipment and the user by enriching the fidelity of the image (see Fig.3). 


\section{Wide collour gamaro for Gollowir LCD with LED-BLU}

1. CCFL spectrum is fixed (Lamp dependent)

2. Each LED spectrum peak can be controlled in real-time

LED's spectrum vs CCFL

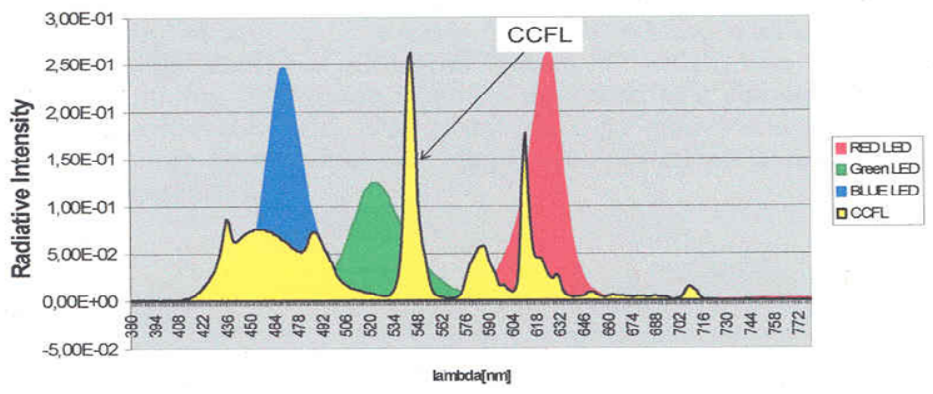

Fig. 3. Wide color gamut of LED backligrhts

\section{LED Backlights in LCD Displays Used for Medical Images}

FIMI Philips developed a novel LED backlight specifically devoted to medical LCD displays. It includes an optical architecture and an overall design concept addressing some weaknesses currently present in the LED backlights solutions available in the market such as the limited luminous efficiency and uniformity.

Medical images visualization needs some specific requirements to be satisfied. The most important of them are:

- Luminance response compliant with the DICOM standard

- High luminance values (> 500 nit)

- Good white and color uniformity

- White point adjustability

- Accurately aligned and stabilized luminance level

- Extended lifetime

LED based backlights allow pursuing most of these goals as explained in the following paragraphs describing the LED BLU developed by FIMI in cooperation with PDS and used for grayscale medical displays.

\subsection{Optical and Mechanical Architecture}

The optical system was derived from a "folded mixing light guide" concept [2] where the optical function is split into two parts: one part for mixing the primary colors into white light and a second part where the light is properly distributed on the LCD glass surface (see Fig. 4). In order to get the desired output light level the system efficiency has to be maximized by reducing as much as possible the light losses along the light path. 
Indeed the length of the mixing light guide has been reduced with respect to the system described in [1] and the additional mirror just in front of the LED was removed.

Since the optimal mixing light guide length is the result of a compromise between good color uniformity (achievable with a long mixing light guide) and minimal light losses (achievable with short light guide), its length was optimized considering the specific requirements of the application.

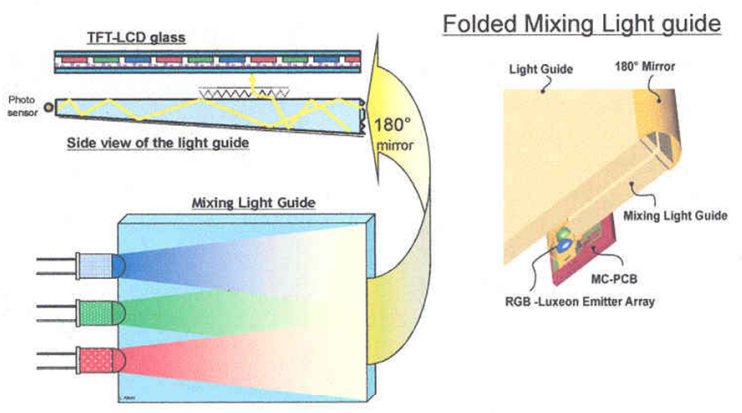

Fig. 4. The optical system of the LED Backlight developed by FIMI

A further light output improvement can be achieved by properly choosing the LED colors. LED are available in fact at several wavelength and flux. In a medical grayscale monitor very saturated colors aren't required - being the images monochromatic - so white LEDs can be included in the LED array. Doing this way some "extra light" can be added at a color temperature close to the target ones.

\subsection{LED Color Mixing}

Though the reasoning described in the paragraph above would let suppose that only white LED is the best solution for what concern the light output level, the color point adjustability asks for colored LED to be included in the array.
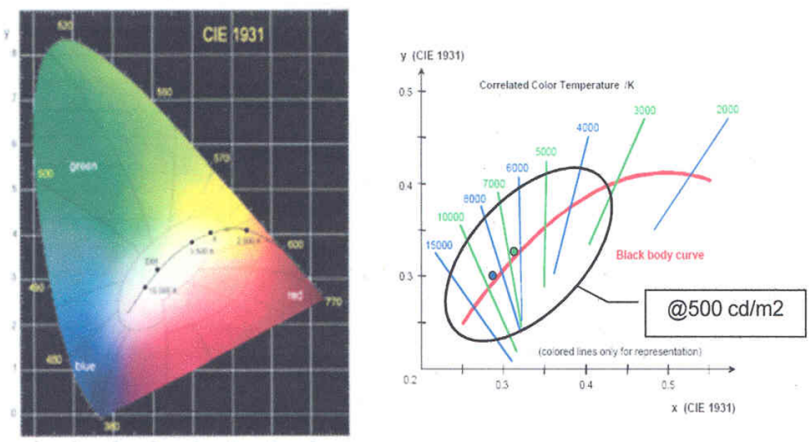

Fig. 5. LEDs enable White Point adjustability unique future in monochrome LCD monitors 
The white point of the monochrome LCD Monitor can be tuned around the black body curve (see Fig. 5).

The reduced color gamut caused by the presence of white LED allows a greater accuracy in controlling each color point inside the gamut itself. Indeed, considering that the LED control is achieved through a PWM driving scheme, the same number of available PWM driving levels is spread on a smaller triangle in the $\mathrm{x}, \mathrm{y}$ chromaticity diagram. This allows a more accurate adjustment and control for each point lying inside the triangle itself.

For the 18 " monitor we used 40 LED's of $1 \mathrm{~W}$ each, binned and matched for a uniform White.

\subsection{Light Loop Control}

The image consistency required by the medical applications needs to keep the light performances of the monitor constant over time.

Besides that, monitors calibrated at the same color temperature must look equal when put one close to the other (e.g. in displays used in the ceiling suspensions of the operating theater)

The former goal can be achieved by keeping the driving level of LED below the maximum at the beginning of the monitor life and increasing the driving level as the LED efficiency decreases due to the aging. The latter requirement can be achieved by continuously and accurately balancing the driving level of each color.

The monitors with CCFL backlight do not allow the color temperature control therefore the display matching can only be achieved by product sorting.

In the implementation described in this paper, both goals are obtained through a closed loop control [1] implemented by a microcontroller that reads the light values supplied by a trichromatic color sensor and accordingly adapts the PWM driving levels.

The graph in the previous Fig. 5 shows the range of color temperature at $500 \mathrm{~cd} / \mathrm{m} 2$ with the present implementation. The optical feedback is shown in Fig. 6.

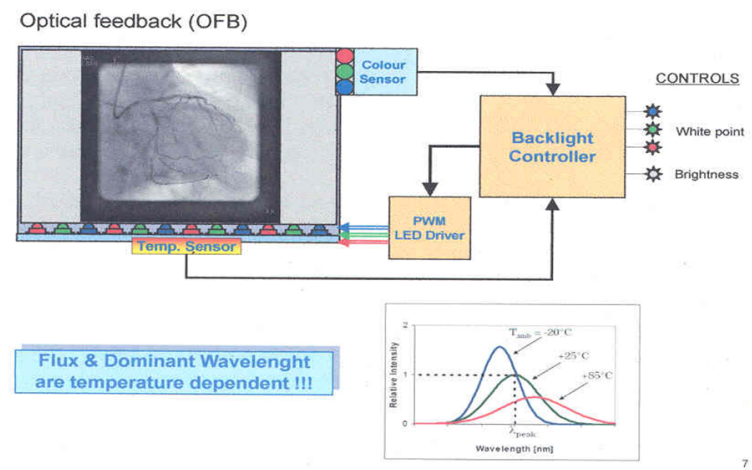

Fig. 6. The optical feedback of the LED backlight 
It gives the benefits of:

- Luminance and colour consistency over time and vs. temperature changes (in fact flux and dominant wavelength are temperature dependent);

- LED aging compensation;

- Accurate white point tuning.

\subsection{Thermal Management}

The LED lifetime is strictly linked to the working temperature of the devices itself: lower working temperatures guarantee longer lifetimes. A good thermal management has to be implemented in order to keep the light output decay as small as possible.

In our design a set of thermal fins has been added on the back of the LED metal core PCB that efficiently spreads the heat produced by the LEDs on a wide surface keeping the devices temperature reasonably low.

Additional cooling systems with axial fans blowing on the mentioned fins could further reduce the working temperature at the expenses of an increased noise level.

\section{Achieved Results}

The previously mentioned techniques have been implemented in monitor samples using array of 40 high power LEDs. Some measurements were carried out in order to evaluate the system performances.

An 18" monochrome monitor was calibrated at three different color temperatures $(6500 \mathrm{~K}, 7600 \mathrm{~K}$ and $9300 \mathrm{~K})$ and at a luminance level of $500 \mathrm{~cd} / \mathrm{m} 2$. The measured power consumption of the complete set was around $49 \mathrm{~W}$. The power consumption was roughly the same for a similar product provided with standard CCFL backlight and calibrated at the same luminance level was. Of course the CCFL model was not adjustable in terms of color temperature.

Further improvements in terms of efficiency are advisable in the near future since the LED performance in terms of lumen per watt is continuously improving.

The brightness and color uniformity of the complete set (including the LCD panel) was evaluated on a standard nine points pattern.

The results were:

- Brightness uniformity $\cong 85 \%$

- Color uniformity $\leq 0.005$ u', v' i.e. matching the typical color detectability limit of the human visual system.

\section{Conclusion}

The LED-based backlight offers the unique feature of controlling the white point of displayed image (fine tuning of the LCD display's white point and maintained over time); it guarantees panel-to-panel consistency in terms of white color and luminance and easier monitor replacement in multi-display installations. 
The current monitors with CCFL backlights do not allow the white point control therefore the display matching can only be obtained through product sorting.

Moreover the developed solution of LED backlight overcomes the main limitations linked to the LED technology such as efficiency and uniformity of both color and brightness and offers saving in cost of ownership by achieving a longer life time

It enhances the image accuracy and stability in critical applications such as the medical imaging ones. In fact appropriate matching of the white point of two or more adjacent displays ensures consistency in the various medical images and improves the accuracy of the task of physicians and radiologists.

\section{Acknowledgements}

The authors thank Mr. Paul Aerssens of Professional Display Systems B.V. for his contribution to the design of the backlight unit.

\section{References}

1. Perduijn, A., et al.: Light Output Feedback Solution for RGB LED Backlight Applications. In: SID 2003 Symposium Digest, vol. 34, pp. 1254-1257

2. Martynov, Y.: High-efficiency Slim LED Backlight System with Mixing Light Guide. In: SID 2003 Symposium Digest, vol. 34, pp. 1259-1261 